\title{
A diffusion approximation for ocean wave scatterings by randomly distributed ice floes
}

\author{
Xin Zhao ${ }^{a, *}$ and Hayley Shen ${ }^{b}$ \\ ${ }^{a}$ Advanced Research Institute for Multidisciplinary Science, Beijing Institute of \\ Technology, Beijing, 100081, China \\ ${ }^{\mathrm{b}}$ Department of Civil and Environmental Engineering, Clarkson University \\ Potsdam, NY, 13699, USA \\ *Corresponding author, Email: xinzhao@ clarkson.edu
}

\begin{abstract}
This study presents a continuum approach using a diffusion approximation method to solve the scattering of ocean waves by randomly distributed ice floes. In order to model both strong and weak scattering, the proposed method decomposes the wave action density function into two parts: the transmitted part and the scattered part. For a given wave direction, the transmitted part of the wave action density is defined as the part of wave action density in the same direction before the scattering; and the scattered part is a first order Fourier series approximation for the directional spreading caused by scattering. An additional approximation is also adopted for simplification, in which the net directional redistribution of wave action by a single scatterer is assumed to be the reflected wave action of a normally incident wave into a semi-infinite ice cover. Other required input includes the mean shear modulus, diameter and thickness of ice floes, and the ice concentration. The directional spreading of wave energy from the diffusion approximation is found to be in reasonable agreement with the previous solution using the Boltzmann equation. The diffusion model provides an alternative method to implement wave scattering into an operational wave model.
\end{abstract}

Keywords: Sea Ice; Wave Scattering; Diffusion Approximation; Directional Spreading 


\section{Introduction}

Propagation of ocean waves into an ice cover is one of many wave phenomena in nature. It shares the same basic conceptual model as in acoustic, elastic, and electromagnetic wave propagation in complex media. The study of ocean waves in ice covered condition has a long history (e.g. Greenhill, 1886). Contemporary studies of this topic have been accelerating due to the rapid decline of ice in the Arctic (Comiso, et al., 2008) and intensified wave activities (Thomson and Rogers, 2014). These conditions combined with increased shipping and environmental concerns call for better models of ocean waves in various ice covers.

As a material, ice covers are extremely inhomogeneous. Their physical properties change dynamically in response to both thermal and mechanical forcing. When ocean waves enter an ice cover, two things may happen: its speed may change and its energy may be reduced/redirected. Two fundamental processes affect the energy: the intrinsic and the scattering attenuation. The first results in net energy loss due to various dissipative processes, many of which have been considered in different models. The second is a reduction of energy in the original wave direction through scattering. The total energy is not affected but only redistributed into other directions. The present study addresses the scattering part of wave propagation.

Scattering is the directional redistribution from the original wave direction (Ishimaru, 1978). Studies at the scatterer scale are the foundation for the macro-scale models, which determine the energy propagation through a large collection of scatterers over a long distance. For instance, Ryzhik et al. (1996) gave the formulation of a general transport equation for wave propagation in random media including both the intrinsic and scattering attenuation. These attenuations from a single scatterer determine the coefficients used in the transport equation. Examples were provided in acoustic, electromagnetic, and elastic waves. Numerous references can be found in each of these fields as listed in their study. 
For ocean waves under ice covers, the scattering process has also been developed from the scatterer scale to the macro-scale. At the scatterer scale, detailed study was conducted for 2-D wave transmission and reflection between open water and ice covers, between ice covers of different properties, where the ice cover was assumed semi-infinite or finite in extent, and for 3-D cases where the ice was circular or arbitrary in shape (reviewed in Squire, 2011). These studies provided the reflection from a single ice boundary in the 2-D case (e.g. Fox and Squire 1990), and the scattering distribution from a single ice floe in the 3-D case (e.g. Meylan and Squire 1996). Utilizing these results, wave propagation through an array of ice floes in 2-D and 3-D cases, with uniform or non-uniform floe sizes and various ice properties have been studied (Dumont et al, 2011; Williams et al., 2013a,b; Doble and Bidlot, 2013; Meylan et al., 1997; Masson and LeBlond, 1989; Meylan and Masson, 2006; Peter et al., 2004; Perrie and Hu, 1996, 1997; Montiel et al., 2016). The goal of these studies is to incorporate the scattering process in an operational ocean model.

There are two approaches used in solving the wave scattering through a large array of ice

floes. One assumes the independence of phase interactions, so that the wave fields generated may be superimposed (e.g. Masson and Leblond, 1989; Meylan et al., 1997). The other solves the multiple-floe domain as a coupled hydroelastic problem with each floe-water interface as part of the complex boundary (Bennetts and Squire, 2009; Montiel et al. 2016). In the present study, we propose a different approach from these two. The proposed method is based on a modified diffusion approximation used in other wave propagation fields. This method is not as accurate as the two approaches mentioned above, but it provides an alternative method which may be easier to incorporate in operational ocean models that need to treat a large variety of ice covers.

\section{The theoretical formulation}

In this section, we derive the governing equations for wave scatterings with a diffusion approximation. The advantage of such approximation is to avoid calculating the complex integral kernel in the integral-differential equation of the wave action density function. 
The diffusion approximation is commonly used in the radiative transfer problem in a random medium (Ryzhik et al. 1996). However, the existing diffusion approximations used in various fields with random scatterers all assume strong scattering, such that the distance over which a single direction wave ray becomes isotropic is short compared with other length scales in a field of scatterers. This assumption allows previous diffusion models to focus on the isotropic part of the wave action density function.

For gravity waves propagating in a field of discrete ice floes, such assumption does not apply well to long waves. We thus propose here a different approach. The general philosophy of this approach is to start with a two-term decomposition for the wave action density function: the transmitted part and the scattered part. The transmitted part attenuates its energy through scatterings. The scattered part gains the energy from the transmitted part and gradually becomes more isotropic. We apply the diffusion approximation for the scattered part to obtain three differential equations. Details of the derivation are given below.

The wave action balance equation of ocean waves is

$$
\frac{\partial}{\partial t} N(\mathbf{x}, t, \mathbf{k})+\nabla \cdot\left[\mathbf{c}_{g} N(\mathbf{x}, t, \mathbf{k})\right]=\frac{S(\mathbf{x}, t, \mathbf{k})}{\omega} .
$$

Here, $N=E / \omega$ is the wave action density function (Andrews and Mcintyre, 1978), in which $E$ is the wave energy density per unit area of angular frequency $\omega, \mathbf{k}$ is the wave number vector, $\mathbf{x}$ is the spatial coordinate, $t$ is time, $\mathbf{c}_{g}$ is the group velocity vector, and $S$ is the total source/sink term. In addition to scattering, the source/sink may include processes such as wind generation, wave breaking, and nonlinear transfer between different frequencies. In the presence of an ice cover, these source/sink terms are not well established. If we ignore all other processes and focus on the scattering process alone, then along each wave component $\mathbf{k}$ the above equation becomes the following Boltzmann equation (Meylan et al., 1997).

$$
\begin{aligned}
& \frac{\partial}{\partial t} N(\mathbf{x}, t, k, \theta)+c_{g} \boldsymbol{\theta} \cdot \nabla N(\mathbf{x}, t, k, \theta)=-c_{g} \alpha_{s}(\mathbf{x}, t, k, \theta) N(\mathbf{x}, t, k, \theta) . \\
& +c_{g} \int_{0}^{2 \pi} S_{k}\left(\mathbf{x}, t, k, \theta, \theta^{\prime}\right) N\left(\mathbf{x}, t, k, \theta^{\prime}\right) d \theta^{\prime}
\end{aligned} .
$$


Here, $\alpha_{s}$ is the scattering attenuation coefficient, $\theta$ indicates the direction of wave number vector $\mathbf{k}, k=|\mathbf{k}|$, and $S_{k}$ is the kernel function of wave energy redistribution. The scattering kernel $S_{k}$ represents wave energy in the $\theta^{\prime}$ direction that is redirected into the $\theta$ direction (Meylan et al. 1997). There has been a considerable amount of study based on thin-elastic-plate theory to derive $S_{k}$ (e.g. Meylan and Squire, 1996; Meylan et al., 1997; and Bennetts and Williams, 2010), which we referred to as the "scatterer scale" studies. The energy conservation condition leads to

$$
\alpha_{s}(\mathbf{x}, t, k, \theta)=\int_{0}^{2 \pi} S_{k}\left(\mathbf{x}, t, k, \theta^{\prime}, \theta\right) d \theta^{\prime} .
$$

Thus, the redistribution of energy is exactly the loss of energy in the given wave direction.

We now propose a decomposition of $N$, which is more effective in following both weak and strong scattering processes. The wave action density function $N$ defined in Eq. (1) is linearly decomposed into two parts,

$$
N(\mathbf{x}, t, k, \theta)=A(\mathbf{x}, t, k, \theta)+B(\mathbf{x}, t, k, \theta) .
$$

We define $A(\mathbf{x}, t, k, \theta)$ as the transmitted part in the direction of $N(\mathbf{x}, t, k, \theta)$, and $B(\mathbf{x}, t, k, \theta)$ is the scattered part. In this decomposition, the amount of the "incident" wave $N$ that remains in the same direction after scattering is isolated from the rest of scattering energy. In this way, we can better treat weakly scattering waves before they become completely isotropic. Furthermore, the evolution of waves from open water, a no scattering region, into an ice field, a scattering region, may also be followed more closely near the boundary between the two regions. Masson and LeBlond (1989) applied a similar decomposition. The governing equations for these two parts are as the following,

$$
\begin{gathered}
\frac{\partial}{\partial t} A(\mathbf{x}, t, k, \theta)+c_{g} \boldsymbol{\theta} \cdot \nabla A(\mathbf{x}, t, k, \theta)=-c_{g} \alpha_{s}(\mathbf{x}, t, k, \theta) A(\mathbf{x}, t, k, \theta), \\
\frac{\partial}{\partial t} B(\mathbf{x}, t, k, \theta)+c_{g} \boldsymbol{\theta} \cdot \nabla B(\mathbf{x}, t, k, \theta)=-c_{g} \alpha_{s}(\mathbf{x}, t, k, \theta) B(\mathbf{x}, t, k, \theta) \\
+c_{g} \int_{0}^{2 \pi} S_{k}\left(\mathbf{x}, t, k, \theta, \theta^{\prime}\right) B\left(\mathbf{x}, t, k, \theta^{\prime}\right) d \theta^{\prime}+c_{g} \int_{0}^{2 \pi} S_{k}\left(\mathbf{x}, t, k, \theta, \theta^{\prime}\right) A\left(\mathbf{x}, t, k, \theta^{\prime}\right) d \theta^{\prime}
\end{gathered}
$$

Equation (5) says energy transmitted in the "incident" wave direction is reduced exactly by the amount of loss from the scattering process. Equation (6) says the scattered energy is increased by contributions of the total redistribution from the scattered part and the 
"incident" part. The governing equation for $A$ is straightforward. To simplify the integral-differential equation for $B$, we adopt a diffusion approximation.

To use the diffusion approximation, we decompose $B$ into a directional averaged part and a fluctuating part as the following,

$$
B(\mathbf{x}, t, k, \theta)=\bar{B}(\mathbf{x}, t, k)+B^{\prime}(\mathbf{x}, t, k, \theta)+\cdots,
$$

where

$$
\bar{B}(\mathbf{x}, t, k)=\frac{1}{2 \pi} \int_{0}^{2 \pi} B(\mathbf{x}, t, k, \theta) d \theta .
$$

Similar to what has been done in previous diffusion approximations, we use a first order approximation for Eq. (7) by including the first two terms in the Fourier series expansion of $B$ with respect to $\theta$ :

$$
B(\mathbf{x}, t, k, \theta)=B_{0}(\mathbf{x}, t, k)+\mathbf{B}_{1}(\mathbf{x}, t, k) \cdot \boldsymbol{\theta}+\cdots .
$$

In the above,

$$
\begin{gathered}
B_{0}(\mathbf{x}, t, k)=\bar{B}(\mathbf{x}, t, k), \\
\mathbf{B}_{1}(\mathbf{x}, t, k) \cdot \boldsymbol{\theta}=B_{1 x}(\mathbf{x}, t, k) \cos \theta+B_{1 y}(\mathbf{x}, t, k) \sin \theta=B^{\prime}(\mathbf{x}, t, k, \theta), \\
B_{1 x}(\mathbf{x}, t, k)=\frac{1}{\pi} \int_{0}^{2 \pi} B(\mathbf{x}, t, k, \theta) \cos \theta d \theta \\
B_{1 y}(\mathbf{x}, t, k)=\frac{1}{\pi} \int_{0}^{2 \pi} B(\mathbf{x}, t, k, \theta) \sin \theta d \theta .
\end{gathered}
$$

We now make two assumptions: 1) a local equilibrium condition for $B^{\prime}$, i.e. $\partial B^{\prime} / \partial t \approx 0$; and 2) $\alpha_{s}$ is independent of $\theta$. The first condition means

$$
\begin{gathered}
\frac{\partial B_{1 x}}{\partial t}=\frac{1}{\pi} \int_{0}^{2 \pi} \frac{\partial B(\mathbf{x}, t, k, \theta)}{\partial t} \cos \theta d \theta \approx 0, \\
\frac{\partial B_{1 y}}{\partial t}=\frac{1}{\pi} \int_{0}^{2 \pi} \frac{\partial B(\mathbf{x}, t, k, \theta)}{\partial t} \sin \theta d \theta \approx 0 .
\end{gathered}
$$

This condition is satisfied when $\frac{\partial B^{\prime}}{\partial t}<<c_{g} \alpha_{s} B^{\prime}$, or $c_{g} \alpha_{s} \Delta t>>1$. The scattering attenuation $\alpha_{s} \approx e / l$, where $l$ is the distance between centers of neighboring ice floes and $e$ is the percent of energy scattered at each floe. The size of the computational grid is 
$\Delta x \approx c_{g, \max } \Delta t$ from Courant-Friedrichs-Lewy stability condition. Using the deep water condition to estimate, we have $c_{g}=g / 2 \omega$ and $c_{g, \max }=g / 2 \omega_{\min }$. The wavelength is $L^{\prime}=2 \pi g / \omega^{2}$ and $L_{\max }^{\prime}=2 \pi g / \omega_{\min }^{2}$. Therefore, $c_{g} \alpha_{s} \Delta t \approx e \frac{\omega}{\omega_{\min }} \frac{L^{\prime}}{l} \frac{\Delta x}{L_{\max }}$. For cells much greater than the wavelength, $c_{g} \alpha_{s} \Delta t>>1$ is satisfied. An isotropic $\alpha_{s}$ implies

$$
\alpha_{s}(\mathbf{x}, t, k, \theta)=\alpha_{s}(\mathbf{x}, t, k)=\int_{0}^{2 \pi} S_{k}\left(\mathbf{x}, t, k, \theta^{\prime}, \theta\right) d \theta^{\prime},
$$

hence

$$
S_{k}\left(\mathbf{x}, t, k, \theta^{\prime}, \theta\right)=S_{k}\left(\mathbf{x}, t, k, \theta^{\prime}\right)
$$

We multiply both sides of Eq. (6) by $\cos \theta$ then integrate over $2 \pi$. The left hand side is approximately zero from assumption 1 . The right hand side becomes, after applying assumption $2, \pi \frac{\partial \bar{B}}{\partial x}+\pi \alpha_{s} B_{1 x}$. We thus obtain

$$
-\alpha_{s} B_{1 x}=\frac{\partial \bar{B}}{\partial x}
$$

Similarly, we repeat the above by multiplying Eq. (6) with $\sin \theta$ to get

$$
-\alpha_{s} B_{1 y}=\frac{\partial \bar{B}}{\partial y} \text {. }
$$

Substituting Eqs. (18) and (19) into Eq. (11), we have

$$
B^{\prime}(\mathbf{x}, t, k, \theta)=-\frac{1}{\alpha_{s}} \theta \cdot \nabla \bar{B}(\mathbf{x}, t, k) .
$$

To obtain the governing equation of $\bar{B}(\mathbf{x}, t, k)$, we take the directional average of Eq. (6), which yields

$$
\begin{aligned}
& \frac{\partial}{\partial t} \bar{B}(\mathbf{x}, t, k)+c_{g} \frac{1}{2 \pi} \int_{0}^{2 \pi} \boldsymbol{\theta} \cdot \nabla B(\mathbf{x}, t, k, \theta) d \theta= \\
& -c_{g} \frac{1}{2 \pi} \int_{0}^{2 \pi} \alpha_{s}(\mathbf{x}, t, k, \theta) B(\mathbf{x}, t, k, \theta) d \theta+c_{g} \frac{1}{2 \pi} \int_{0}^{2 \pi} \int_{0}^{2 \pi} S_{k}\left(\mathbf{x}, t, k, \theta, \theta^{\prime}\right) d \theta B\left(\mathbf{x}, t, k, \theta^{\prime}\right) d \theta^{\prime} . \\
& +c_{g} \frac{1}{2 \pi} \int_{0}^{2 \pi} \int_{0}^{2 \pi} S_{k}\left(\mathbf{x}, t, k, \theta, \theta^{\prime}\right) d \theta A\left(\mathbf{x}, t, k, \theta^{\prime}\right) d \theta^{\prime}
\end{aligned}
$$

Using the energy conservation condition Eq. (3), we obtain 


$$
\begin{aligned}
& \frac{\partial}{\partial t} \bar{B}(\mathbf{x}, t, k)+c_{g} \frac{1}{2 \pi} \int_{0}^{2 \pi} \boldsymbol{\theta} \cdot \nabla B(\mathbf{x}, t, k, \theta) d \theta= \\
& -c_{g} \frac{1}{2 \pi} \int_{0}^{2 \pi} \alpha_{s}(\mathbf{x}, t, k, \theta) B(\mathbf{x}, t, k, \theta) d \theta+c_{g} \frac{1}{2 \pi} \int_{0}^{2 \pi} \alpha_{s}\left(\mathbf{x}, t, k, \theta^{\prime}\right) B\left(\mathbf{x}, t, k, \theta^{\prime}\right) d \theta^{\prime} .(22) \\
& +c_{g} \frac{1}{2 \pi} \int_{0}^{2 \pi} \alpha_{s}\left(\mathbf{x}, t, k, \theta^{\prime}\right) A\left(\mathbf{x}, t, k, \theta^{\prime}\right) d \theta^{\prime}
\end{aligned}
$$

Eliminating the first and second terms on the right hand side of the above equation, we get

$$
\frac{\partial}{\partial t} \bar{B}(\mathbf{x}, t, k)+c_{g} \frac{1}{2 \pi} \int_{0}^{2 \pi} \boldsymbol{\theta} \cdot \nabla B(\mathbf{x}, t, k, \theta) d \theta=c_{g} \frac{1}{2 \pi} \int_{0}^{2 \pi} \alpha_{s}\left(\mathbf{x}, t, k, \theta^{\prime}\right) A\left(\mathbf{x}, t, k, \theta^{\prime}\right) d \theta^{\prime} .
$$

For the second term of the left hand side in the above equation, we substitute Eqs. (7) and (20) into it to get

$$
\begin{aligned}
& \frac{1}{2 \pi} \int_{0}^{2 \pi} \boldsymbol{\theta} \cdot \nabla B(\mathbf{x}, t, k, \theta) d \theta=\frac{1}{2 \pi}\left(\int_{0}^{2 \pi} \cos \theta d \theta \frac{\partial}{\partial x} \bar{B}(\mathbf{x}, t, k)+\int_{0}^{2 \pi} \sin \theta d \theta \frac{\partial}{\partial y} \bar{B}(\mathbf{x}, t, k)\right) \\
& -\frac{1}{2 \pi} \int_{0}^{2 \pi} \boldsymbol{\theta} \cdot \nabla \frac{1}{\alpha_{s}} \theta \cdot \nabla \bar{B}(\mathbf{x}, t, k) d \theta
\end{aligned}
$$

The two terms in the parentheses equal to zero. Hence,

$$
\frac{1}{2 \pi} \int_{0}^{2 \pi} \boldsymbol{\theta} \cdot \nabla B(\mathbf{x}, t, k, \theta) d \theta=-\frac{1}{2 \pi} \int_{0}^{2 \pi} \boldsymbol{\theta} \cdot \nabla \frac{1}{\alpha_{s}} \boldsymbol{\theta} \cdot \nabla \bar{B}(\mathbf{x}, t, k) d \theta .
$$

After some algebra, we obtain

$$
\frac{1}{2 \pi} \int_{0}^{2 \pi} \boldsymbol{\theta} \cdot \nabla \frac{1}{\alpha_{s}} \boldsymbol{\theta} \cdot \nabla \bar{B}(\mathbf{x}, t, k) d \theta=\nabla \cdot \mathbf{D}(\mathbf{x}, t, k) \cdot \nabla \bar{B}(\mathbf{x}, t, k) .
$$

Since we assumed that $\alpha_{s}$ is isotropic,

$$
\mathbf{D}(\mathbf{x}, t, k)=\frac{c_{g}}{2 \alpha_{s}(\mathbf{x}, t, k)} \mathbf{I} .
$$

Here, $\mathbf{I}$ is the unit tensor. Substituting the above into Eq. (23), we get

$$
\frac{\partial}{\partial t} \bar{B}(\mathbf{x}, t, k)=\nabla \cdot \mathbf{D}(\mathbf{x}, t, k) \cdot \nabla \bar{B}(\mathbf{x}, t, k)+c_{g} \alpha_{s}(\mathbf{x}, t, k) \frac{1}{2 \pi} \int_{0}^{2 \pi} A\left(\mathbf{x}, t, k, \theta^{\prime}\right) d \theta^{\prime} .
$$

In summary, we obtain the governing equations for the energy action density function as the following,

$$
N(\mathbf{x}, t, k, \theta)=A(\mathbf{x}, t, k, \theta)+\bar{B}(\mathbf{x}, t, k)+B^{\prime}(\mathbf{x}, t, k, \theta),
$$




$$
\begin{gathered}
\frac{\partial}{\partial t} A(\mathbf{x}, t, k, \theta)+c_{g} \boldsymbol{\theta} \cdot \nabla A(\mathbf{x}, t, k, \theta)=-c_{g} \alpha_{s}(\mathbf{x}, t, k) A(\mathbf{x}, t, k, \theta) \\
\frac{\partial}{\partial t} \bar{B}(\mathbf{x}, t, k)=\nabla \cdot \mathbf{D}(\mathbf{x}, t, k) \cdot \nabla \bar{B}(\mathbf{x}, t, k)+c_{g} \alpha_{s}(\mathbf{x}, t, k) \frac{1}{2 \pi} \int_{0}^{2 \pi} A\left(\mathbf{x}, t, k, \theta^{\prime}\right) d \theta^{\prime} \\
B^{\prime}(\mathbf{x}, t, k, \theta)=-\frac{1}{\alpha_{s}} \mathbf{\theta} \cdot \nabla \bar{B}(\mathbf{x}, t, k)
\end{gathered}
$$

Although Eq. (31) has an integral-differential form, because $A$ is already solved in Eq. (30), Eq. (31) is again a simple diffusion equation.

\section{The scattering attenuation}

Before we apply this diffusion model, we need to evaluate the scattering attenuation coefficient $\alpha_{s}$ given in Eq. (3), which requires the solution of a 3-D problem such as in Meylan and Squire (1996) for a circular floe, or in Bennetts and Williams (2010) for an arbitrary shape floe. Here we propose a simpler method, hence an approximation, based on a 2-D wave-ice interaction theory (Wadhams, 1986). We begin by considering the discrete version for the evolution equation of the wave action density function.

$$
A(\mathbf{x}, t+\Delta t, k, \theta)-A(\mathbf{x}, t, k, \theta)=-c_{g} \theta \cdot \nabla A(\mathbf{x}, t, k, \theta) \Delta t-r A(\mathbf{x}, t, k, \theta) .
$$

Here, $r$ is the energy loss coefficient from the incident wave. In a two dimensional model, scattered waves can only go to the direction opposite to the incident wave. In this case $r=R^{2}, R$ is the wave reflection coefficient by a single ice edge of a prescribed thickness. This equation assumes that in $\Delta t$ there is only one wave reflection event. Allowing for multiple reflections between floes, the total energy loss $\Delta A$ in $\Delta t$ is (Wadhams, 1986)

$$
\Delta A(\mathbf{x}, t, k, \theta)=-\sum_{i=1}^{M} r(1-r)^{i-1} A(\mathbf{x}, t, k, \theta)=-\left(1-(1-r)^{M}\right) A(\mathbf{x}, t, k, \theta) .
$$

Here, $M(\Delta t)=I N T(\Delta t / \sigma)$, the closest integer $\leq \Delta t / \sigma, \sigma=l / c_{g}$ is the characteristic time for one wave reflection event, $l=D / c$ is the distance between the centers of two 
ice floes. Here, $D$ is the width of ice floes, and $c$ is the ice concentration. Equation (33) thus becomes

$$
A(\mathbf{x}, t+\Delta t, k, \theta)-A(\mathbf{x}, t, k, \theta)=-c_{g} \theta \cdot \nabla A(\mathbf{x}, t, k, \theta) \Delta t-\left(1-(1-r)^{c_{g} \Delta t / l}\right) A(\mathbf{x}, t, k, \theta) .
$$

Dividing the above equation by $\Delta t$,

$$
\frac{A(\mathbf{x}, t+\Delta t, k, \theta)-A(\mathbf{x}, t, k, \theta)}{\Delta t}=-c_{g} \theta \cdot \nabla A(\mathbf{x}, t, k, \theta)-\frac{\left(1-(1-r)^{c_{g} \Delta t / l}\right)}{\Delta t} A(\mathbf{x}, t, k, \theta) .
$$

Let $\Delta t \rightarrow 0$,

$$
\frac{\partial A(\mathbf{x}, t, k, \theta)}{\partial t}=-c_{g} \theta \cdot \nabla A(\mathbf{x}, t, k, \theta)+\frac{c_{g}}{l} \ln (1-r) A(\mathbf{x}, t, k, \theta) .
$$

From the above, we have the temporal decay coefficient

$$
\alpha_{s}^{\prime}=-\frac{c_{g}}{l} \ln (1-r) .
$$

The spatial scattering coefficient is thus

$$
\alpha_{s}=-\frac{1}{l} \ln (1-r) .
$$

This result is the same as in Wadhams (1986) and Bennetts and Squire (2012). With $\alpha_{s}$ determined, Eqs. (30-32) form a closed system.

To obtain $r$ we follow the method developed in Zhao and Shen (2015). In which the reflection coefficient is solved assuming a wave propagating from open water into a semi-infinite elastic sheet of finite thickness. The velocities and stresses at the vertical interface between the open water and the ice cover, and between water bodies on the open water side and the ice covered side are matched using the variational method. The solution is obtained by including 20 evanescent modes in addition to the two propagating modes.

\section{Validation of the diffusion solution}

To validate the simplified scattering model based on the proposed diffusion approach, we consider a case as described in Meylan et al. (1997) where no wave damping except scattering is considered. A monochromatic wave enters an ice floe field as shown in Fig. 
1. The ice concentration is 0.5 , the ice thickness is $1 \mathrm{~m}$, the Young's modulus is $6 \mathrm{GPa}$, Poisson ratio is 0.3 and the diameter of the circular ice floes is $50 \mathrm{~m}$. The wave period is $10 \mathrm{~s}$. The incident wave from the left has a narrow directional distribution described by $\cos ^{20} \theta$ for $-\pi / 2 \leq \theta<\pi / 2$. We reproduce the result of Meylan et al. (1997) from solving Eq. (2) to compare with those from the diffusion approximation described above.

The results for the diffusion approximation are simulated using Eqs. (30-32). The left boundary condition for $A$ is the fixed boundary condition,

$$
A(x=0, t, k, \theta)=\cos ^{20} \theta, \text { for }-\pi / 2 \leq \theta<\pi / 2 .
$$

This boundary condition says that the incident wave at the ice edge is constant in time. The right boundary condition for $A$ is the free boundary condition.

$$
A(x=L, t, k, \theta)=A(x=L-\Delta x, t, k, \theta) .
$$

This boundary condition says that there is no energy reflected at the right, hence it simulates a semi-infinite ice cover. Here, $L$ is the length of the computational domain, and $\Delta x$ is the grid spacing. The right boundary condition for scattered waves $\bar{B}$ is also the free boundary condition.

$$
\bar{B}(x=L, t, k)=\bar{B}(x=L-\Delta x, t, k) .
$$

To derive the boundary condition at the ice edge $(x=0)$ for $\bar{B}$, we use a method commonly adopted in optics (Wang and $\mathrm{Wu}, 2007)$. We extrapolate $\bar{B}(x, t, k)$ to a new location proportional to the characteristic diffusion length $1 / \alpha_{s}$ and set $\bar{B}\left(x=-1 /\left(C \alpha_{s}\right), t, k\right)=0$. Here, $C$ is a nondimensional parameter. Using Taylor expansion to first order, we obtain the boundary condition as the following,

$$
\bar{B}\left(x=-1 /\left(C \alpha_{s}\right), t, k\right)=\bar{B}(x=0, t, k)-\frac{1}{C \alpha_{s}} \frac{\partial \bar{B}(x=0, t, k)}{\partial x}=0,
$$

or

$$
C \alpha_{s} \bar{B}(x=0, t, k)=\frac{\partial \bar{B}(x=0, t, k)}{\partial x} .
$$

Wang and $\mathrm{Wu}$ (2007) showed that $C=1$ for the refractive index matched case. 
In the case study, the computational domain is $100 \mathrm{~km}$ long. Time step $\Delta t=10 \mathrm{~s}, \Delta x=1$ $\mathrm{km}$ in this case study. In Meylan et al. (1997), they solved the steady state solution for Eq. (2). For comparison, we integrate Eqs. (30-32) for 10 hours. At which time, in the computational domain the relative change of the solutions everywhere is less than $10^{-3}$. Fig. 2(a) shows the comparison of the directional spectrum of the wave action density function for waves propagating into the ice field at $10 \mathrm{~km}$. Fig. 2(b) is for $50 \mathrm{~km}$. We see that the diffusion approximation method obtains similar patterns compared with the results from Meylan et al. (1997) (with a correction as mentioned in Meylan (2000)). In Fig. 3, we show the contributions of each term to the whole wave action density function in the diffusion approximation method. It is seen that the $\operatorname{transmitted} \operatorname{part} A \operatorname{decreases}$ along the propagation direction, while the scattered part $\bar{B}$ gains energy from $A$. The distribution of $B^{\prime}$ is anisotropic, as a result of the anisotropic spatial gradient of $\bar{B}$. In Fig. 4, the $8 \mathrm{~s}$ case is added as an additional check. We can find that the directional spectrum becomes isotropic at $10 \mathrm{~km}$ and $50 \mathrm{~km}$. The results from the diffusion approximation agree with the results from the full Boltzmann equation method.

\section{Discussions and conclusions}

In the above, a diffusion approximation method is proposed to simulate wave scattering due to randomly distributed ice floes. This method requires only the mean values of the ice cover: average ice thickness, concentration, mechanical properties and diameter of ice floes. This diffusion approximation method decomposes the wave action density function into the transmitted part and the scattered part. The scattered part is approximated by a first order Fourier series. Two assumptions are made to close the system of equations. They are 1) the local equilibrium condition for $B^{\prime}$, i.e. $c_{g} \alpha_{s} \Delta t>>1$; and 2) $\alpha_{s}$ is isotropic.

The results from the diffusion approximation are shown to agree reasonably well with those from the Boltzmann equation under the same wave and ice conditions for a $10 \mathrm{~s}$ wave. As an additional check, an $8 \mathrm{~s}$ wave is also tested under the same ice condition. 
The results are shown in Fig. 4. Again the Boltzmann equation solution and the diffusion approximation are close to each other. Both show stronger energy redistribution than the 10 s wave.

When applying the diffusion approximation, the scattering coefficient $\alpha_{s}$ is a critical parameter to get accurate solutions. In section 3, we provided an approximate method for this coefficient using a 2-D method. For the 2-D case, the resulting $\alpha_{s}$ is shown in Eq. (39). The 3-D solution of Eq. (3) needs the kernel function $S_{k}$ whose derivation can be found in Meylan et al. (1997). Here we test the validity of this approximation by examining a case of scattering from an ice floe of diameter $50 \mathrm{~m}$, thickness $1 \mathrm{~m}$, Young's modulus $6 \mathrm{GPa}$, Poisson ration 0.3 , and wave period $10 \mathrm{~s}$. We reproduce the results given in Meylan et al. (1997). We then calculate the 2-D results using Eq. (39). As shown in Fig. 5, the scattering coefficients obtained by these two methods are in reasonable agreement except at low periods, where the 2-D scattering attenuation is significantly lower than the 3-D counterpart. The 2-D solution appears to be non-monotonic with respect to wave period. The dip surrounding the period of $14 \mathrm{~s}$ is most likely a numerical artifact. A fifth degree polynomial fit for the 2-D results show good approximation of the 3-D results. The behaviors of the attenuations in Fig. 5 are different from the results in Perrie and $\mathrm{Hu}$ (1996), which include wind generation, wave breaking, nonlinear wavewave interactions, and the motions of the ice floes, in addition to scattering. By including these additional effects, the wave attenuation displayed a roll-over phenomenon observed in the field (Wadhams et al. 1988).

To implement the diffusion approximation proposed in this study into an operational wave model, we envision the following. Suppose that the wave spectrum at some instant over the whole region of concern is known. We now need to update the next time step wave spectrum over the whole region. At each computation grid, we need to first determine the modified spectrum due to the other processes except the scattering, using Eq. (2) with the right hand side including all other source and sink terms due to floe interaction among themselves and floe-water interactions, nonlinear wave-wave interactions between different frequencies, wind input, and wave breaking dissipation. 
The time step for the processes excluding scattering is called the "outer time step". The result would be the wave action $N(\mathbf{x}, t, \mathbf{k})$ without the scattering effect. We then determine how the scattering process would redistribute this $N(\mathbf{x}, t, \mathbf{k})$ in each computational grid during the outer time step. To do so, an iterative method is performed using Eqs. (30-32). Initially, we set $A=N$, and $\bar{B}=B^{\prime}=0$. Integrating Eqs. (30-32) for several sub time steps we get updated $A, \bar{B}$, and $B^{\prime}$. The updated spectrum is obtained by summing up the three terms $N=A+\bar{B}+B^{\prime}$ to obtain the new $N$ for the next iteration. The accuracy of the diffusion solution depends on the diffusion time step, which is a numerical issue that will require careful study on its own.

In conclusion, this study provides an alternative method to model the wave scattering process in ice covered ocean. Details of the scattering distribution are simplified into an isotropic scattering attenuation coefficient. The scattering process is simplified into a diffusion process by assuming local equilibrium of the scattered quantity. Through separating the wave field into the transmitted part and the scattered part, this model can follow the weakly scatter waves, which was not possible in existing diffusion models for wave scattering processes.

The proposed diffusion model at present is still crude. There are several needed studies to improve the proposed model:

1. A better estimate of the diffusion attenuation coefficient.

2. A thorough numerical study of the accuracy of the diffusion approximation.

3. Comparisons on the efficiencies of the diffusion model and the Boltzmann equation approach in operational wave models.

And finally, extending the current model for anisotropic diffusion would also expand the utility of the model to different ice cover conditions.

\section{Acknowledgement}

This work is supported in part by the Office of Naval Research Grant \#N00014-13-10294 and AcRF Tier 2 Grant No. MOE2013-T2-1-054, Ministry of Education, Singapore. 
The authors are grateful for thoughtful comments from the two anonymous reviewers, as well as discussions with Mr. Sukun Cheng of the Clarkson University. This study was conducted when the first author was a postdoctoral fellow at Clarkson University.

\section{References}

Andrews, D. G. and Mcintyre, M. E. 1978. On wave-action and its relatives. J. Fluid Mech., 89(4), 647-664.

Bennetts, L. G. and Squire, V. A. 2009 Wave scattering by multiple rows of circular ice floes. Journal of Fluid Mechanics, 639, 213-238.

Bennetts, L.G. and Squire, V.A., 2012. On the calculation of an attenuation coefficient for transects of ice-covered ocean. Proc. R. Soc. A, 468, No. 2137, pp. 136-162.

Bennetts, L.G. and Williams, T.D., 2010. Wave scattering by ice floes and polynyas of arbitrary shape. Journal of Fluid Mechanics, 662, 5-35.

Comiso, J.C., Parkinson, C.L., Gersten, R. and Stock, L., 2008. Accelerated decline in the Arctic sea ice cover. Geophysical Research Letters, 35, L01703.

Doble, M. J. and Bidlot, J. R., 2013. Wave buoy measurements at the Antarctic sea ice edge compared with an enhanced ECMWF WAM: Progress towards global waves-in-ice modelling. Ocean Modelling, 70, 166-173.

Dumont, D., Kohout, A. and Bertino, L., 2011. A wave-based model for the marginal ice zone including a floe breaking parameterization. Journal of Geophysical Research: Oceans, $116(\mathrm{C} 4)$.

Fox, C., and Squire, V.A., 1990. Reflection and transmission characteristics at the edge of shore fast sea ice. Journal of Geophysical Research: Oceans, 95(C7), 11629-11639. 
Greenhill, A.G., 1886. Wave motion in hydrodynamics. American Journal of Mathematics, 9(1), 62-96.

Ishimaru, A., 1978. Wave Propagation and Scattering in Random Media. Academic Press.

Masson, D., and LeBlond, P., 1989. Spectral evolution of wind-generated surface gravity waves in a dispersed ice field. J. Fluid Mech. 202, 111-136.

Meylan, M.H., 2000. Wave scattering in the marginal ice zone. In A. J. Pullan and D. M. Ryan, eds., ANZIAM 2000, The 36th Applied Mathematics Conference, Copthorne Resort, Waitangi, Bay of Islands, New Zealand, 8-12 February 2000, ISB Number 7259 0869 6. Auckland, Australian Mathematical Society, 43.

Meylan, M.H., 2002. Wave response of an ice floe of arbitrary geometry. Journal of Geophysical Research (Oceans), 107, 3005.

Meylan, M. H., and Masson, D., 2006. A Linear Boltzmann Equation to Model Wave Scattering in the Marginal Ice Zone. Ocean Modelling, 11(3-4), 417-427.

Meylan, M. H., and Squire, V. A., 1996. Response of a circular ice floe to ocean waves. J. Geophys. Res. 101 (C4), 8869-8884.

Meylan, M. H., Squire, V. A., and Fox, C., 1997. Towards realism in modeling ocean wave behavior in marginal ice zones. J. Geophys. Res. 102 (C10), 22981-22991.

Montiel, F., Squire, V.A., and Bennetts, L.G., 2016. Attenuation and directional spreading of ocean wave spectra in the marginal ice zone. Journal of Fluid Mechanics, $790,492-522$. 
Perrie, W. and Hu, Y., 1996. Air-ice-ocean momentum exchange. Part 1: Energy transfer between waves and ice floes. Journal of Physical Oceanography, 26(9), 1705-1720.

Perrie, W. and Hu, Y., 1997. Air-ice-ocean momentum exchange. Part II: Ice drift. Journal of Physical Oceanography, 27(9), 1976-1996.

Peter, M.A., Meylan, M.H., and Chung, H., 2004. Wave scattering by a circular elastic plate in water of finite depth: a closed form solution. International Journal of Offshore and Polar Engineering, 14 (02), 81-85.

Ryzhik, L., Papanicolaou, G., and Keller, J. B., 1996. Transport equations for elastic and other waves in random media. Wave motion, 24 (4), 327-370.

Squire, V. A., 2011. Past, present and impendent hydroelastic challenges in the polar and subpolar seas. Phil. Trans. R. Soc. Lond. A 369, 2813-2831.

Thomson, J. and Rogers, W.E., 2014. Swell and sea in the emerging Arctic Ocean. Geophysical Research Letters, 41(9), 3136-3140.

Wadhams, P., 1986. The seasonal ice zone. In The geophysics of sea ice, 825-991. Springer.

Wadhams, P., V. A. Squire, D. J. Goodman, A. M. Cowan, and S. C. Moore (1988), The Attenuation Rates of Ocean Waves in the Marginal Ice Zone, J. Geophys. Res., 93(C6), 6799-6818.

Wang, L. V., and Wu, H. I., 2007. Biomedical optics: principles and imaging. John Wiley $\&$ Sons. 
Williams, T.D., Bennetts, L.G., Squire, V.A., Dumont, D. and Bertino, L., 2013a. Waveice interactions in the marginal ice zone. Part 1: Theoretical foundations. Ocean Modelling, 71, 81-91.

Williams, T.D., Bennetts, L.G., Squire, V.A., Dumont, D. and Bertino, L., 2013b. Waveice interactions in the marginal ice zone. Part 2: Numerical implementation and sensitivity studies along 1D transects of the ocean surface. Ocean Modelling, 71, 92-101.

Zhao, X. and Shen, H. H., 2015. Ocean wave transmission and reflection by viscoelastic ice covers. Ocean Modelling, 92, 1-10. 


\section{Figures}

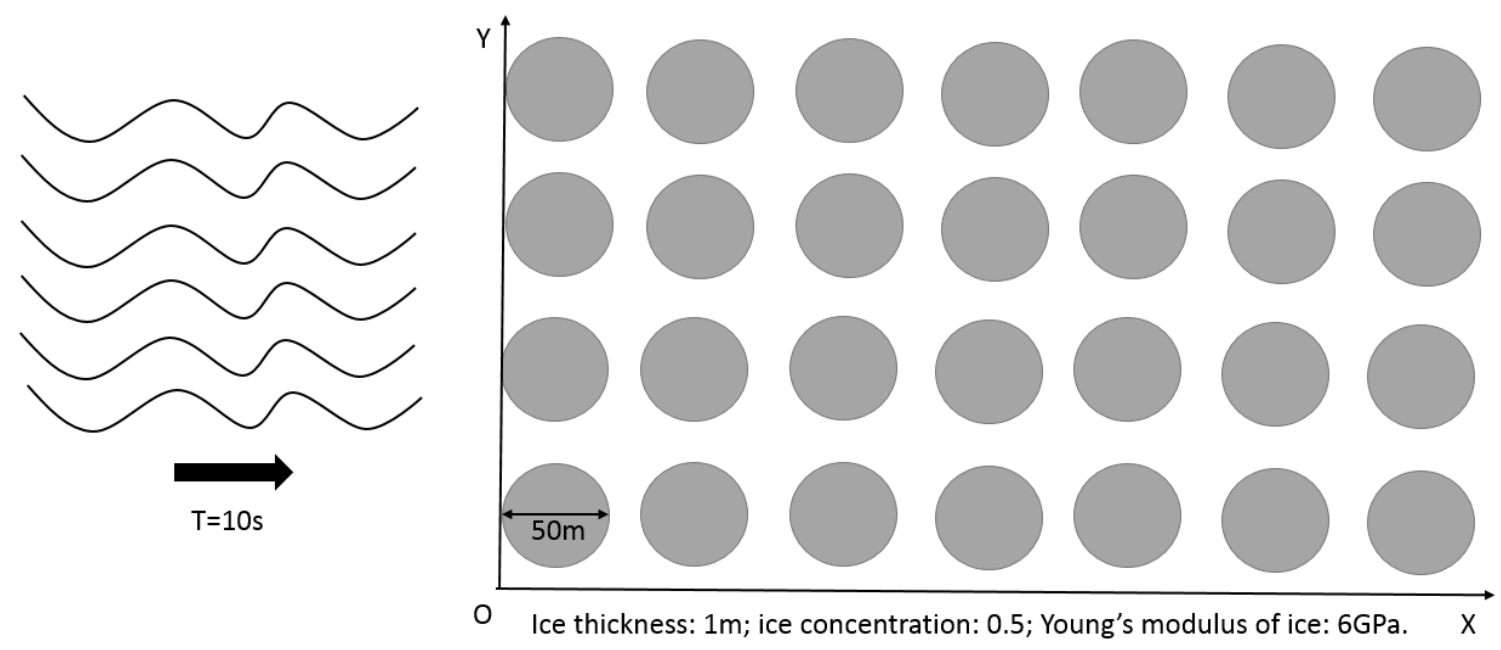

Fig. 1. Schematic of the case.
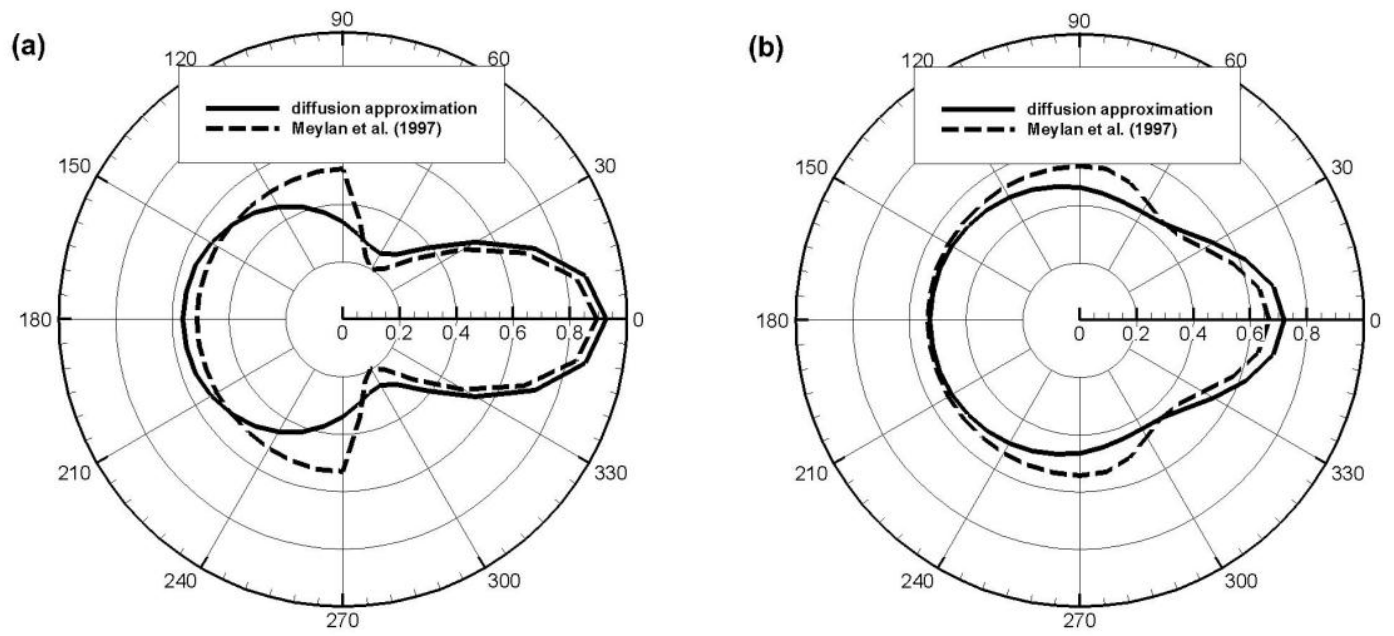

Fig. 2. Comparison on the directional distribution of the wave action density function between diffusion approximation method and the method from Meylan et al. (1997) (with correction given in Meylan (2000)) for the case in Fig. 1 at (a) $x=10 \mathrm{~km}$; (b) $\mathrm{x}=50 \mathrm{~km}$. 

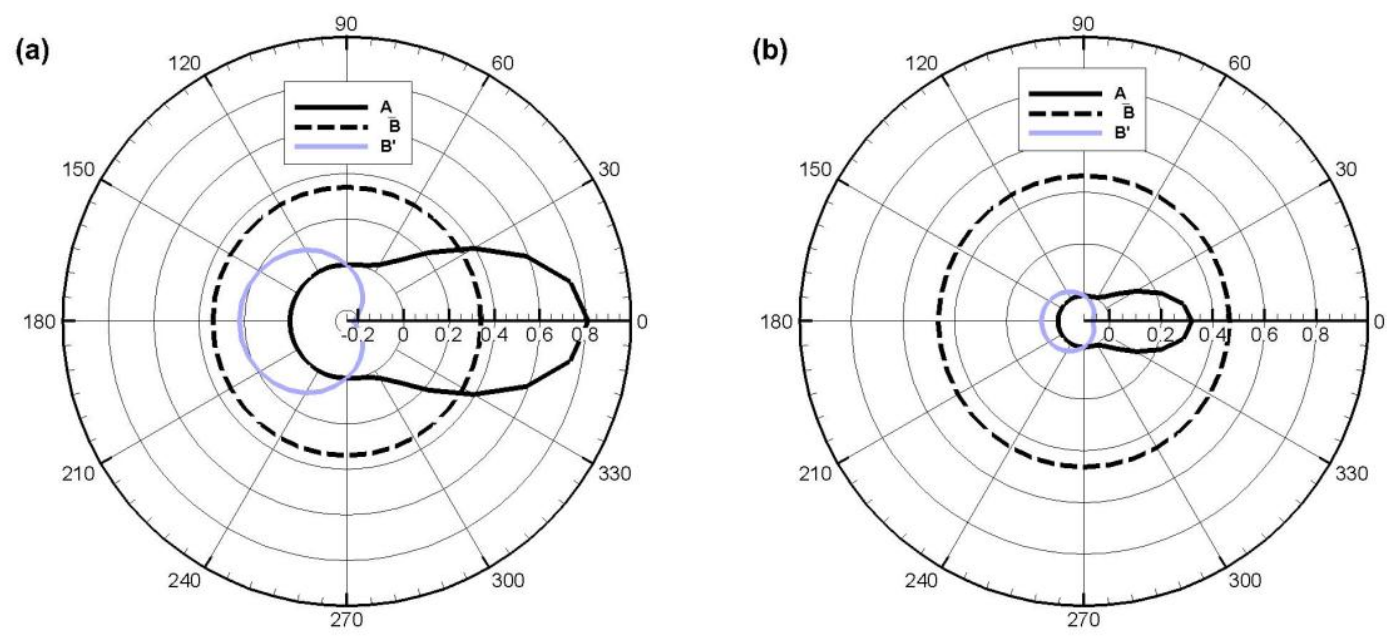

Fig. 3. $A, \bar{B}$, and $B^{\prime}$ from diffusion approximation method for the case in Fig. 1 at (a) $x=10 \mathrm{~km}$; (b) $x=50 \mathrm{~km}$.

(a)

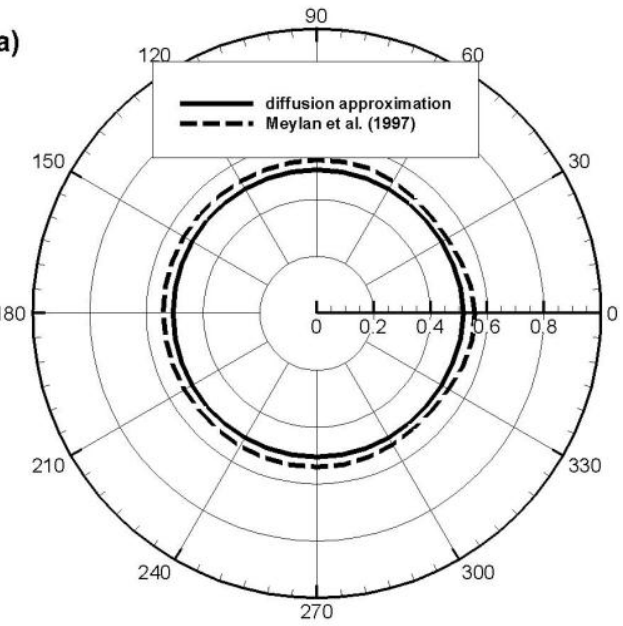

(b)

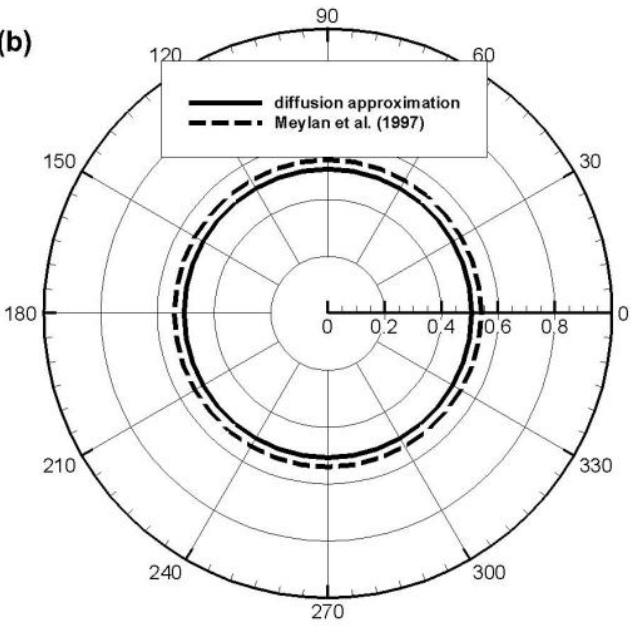

Fig. 4. The same as Fig. 2 except $\mathrm{T}=8 \mathrm{~s}$. 


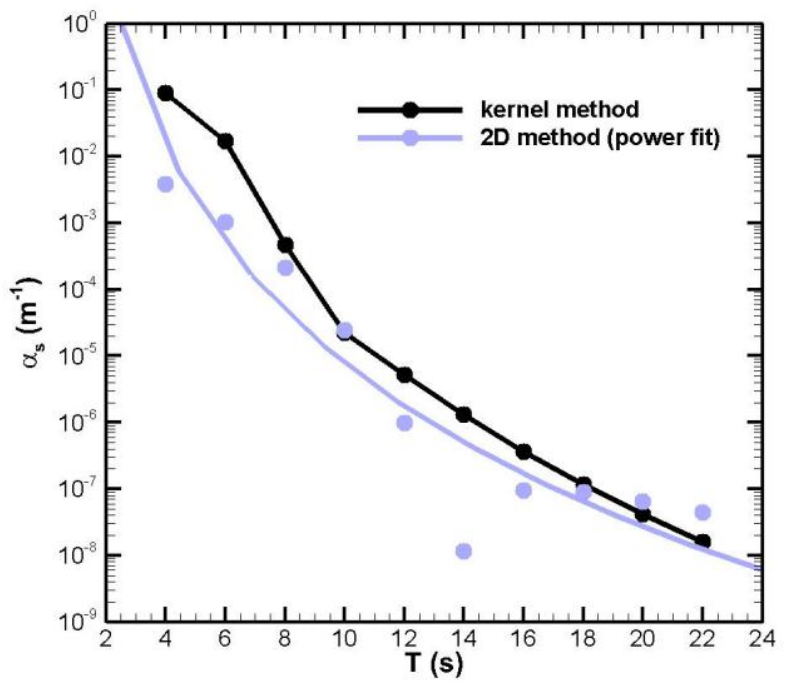

Fig. 5. Comparison on scattering attenuation between the 3-D kernel method and 2-D method. Ice thickness is $1 \mathrm{~m}$, ice concentration is 0.5 , Young's modulus is $6 \mathrm{GPa}$, Poisson's ratio 0.3 , ice floe width in 2-D and ice floe diameter in 3-D are $50 \mathrm{~m}$. For the 2-D method, the water depth of $100 \mathrm{~m}$ and $1000 \mathrm{~m}$ are both tested with nearly identical results. 